\title{
Real-Time Modal Analysis via Wavelength- Swept Spatial and Spectral $\left(\mathrm{S}^{2}\right)$ Imaging
}

\author{
David R. Gray, Marco N. Petrovich, Seyed Reza Sandoghchi, Natalie V. Wheeler, Naveen K. \\ Baddela, Greg T. Jasion, Tom Bradley, David J. Richardson and Francesco Poletti
}

\begin{abstract}
We present a fast implementation of the spatial and spectral imaging $\left(\mathrm{S}^{2}\right)$ technique for modal analysis of multimode optical fibers. It utilizes a continuously scanning tunable laser source with an InGaAs camera operating at $500 \mathrm{~Hz}$ along with inline data processing to increase the measurement repetition rate, by reducing the run up/run down time between two successive scans (about 27 times faster as compared to the previous state of the art). This allows real-time mode content monitoring of a multimode fiber. We illustrate the potential of this tool by collecting an 110,000 wavelength $S^{2}$ spectrogram of a 5 mode-group fiber in minutes, and tracking, in real-time, the four $\mathbf{L P}_{11}$ modes of the same fiber as the launch polarization is rotated.
\end{abstract}

Index Terms - Characterization, Fiber optics, Multimode

\section{INTRODUCTION}

$\mathrm{T}$ HE development of special multimode fibers (MMFs) with the ability to support a discrete number of optical modes/mode groups has recently grown into a topic of great interest due to their potential as next-generation high-capacity transmission fibers enabling spatial division multiplexing (SDM) [1], as well as their application in fiber lasers (where both active fibers and delivery fibers are often few-moded by design to access large mode areas - but operated effectively in an single mode regime [2]), and for optical fiber sensing [3].

In order to support such a fast-growing fiber development effort, several modal content characterization techniques have been proposed and investigated in recent years for measuring MMFs and associated fiber devices [4-6]. Amongst them, the self-interferometric technique based on spatial and spectral $\left(\mathrm{S}^{2}\right)$ imaging has established itself as a powerful and widely adopted tool, due to its high sensitivity (i.e. the ability to detect very small power fractions in higher order modes) and the relative ease of implementation since it does not require a dedicated external interferometer. The $\mathrm{S}^{2}$ technique, originally developed

Manuscript recived month day, 2015; revised month day, year; accepted month day. Date of publication month day; date of current version month day. This work was supported in part by the EU 7th Framework Programme under grant agreement 228033 (MODE-GAP) and UK EPSRC through grant EP/H02607X/1 (EPSRC Centre for Advanced Manufacturing in Photonics)

The authors are with the Optoelectronics Research Centre, University of Southampton, Southampton SO17 1BJ, U.K. (e-mail: drg1v07@ soton.ac.uk;


nkb1c10@orc.soton.ac.uk; g.jasion@soton.ac.uk; T.Bradley@soton.ac.uk; djr@orc.soton.ac.uk; frap@orc.soton.ac.uk).



Fig. 1. Schematic (a) of the wavelength-swept $\mathrm{S}^{2}$ measurement setup, highlighting the direct triggering of the CCD via the TLS and real time data analysis capability, input and output polarization control (IPC and OPC respectively) (b) The triggering system only collects data in the linear part of the TLS scan. For a $500 \mathrm{pm} / \mathrm{s}$ scan speed and a $500 \mathrm{~Hz}$ frame rate, a real time, $\sim 1 \mathrm{~s}$ measurement, can be made with 400 wavelengths captured in $\sim 0.8 \mathrm{~s}$.

by Nicholson et al. [7], spatially resolves the interference that arises at the fiber output due to the different group velocity of the various optical modes. As shown in Fig.1(a)(inset), $\mathrm{S}^{2}$ provides for each guided fiber mode the relative intensity (MPI) and differential group delay (DGD) compared to a dominant mode (often, but not always, the fundamental mode (FM)), as well as the intensity profile and phase of its electric field. Most importantly it does not require any prior knowledge about the fiber under test (FUT).

The fastest $S^{2}$ data collection system to date is commercially available from Interfiber Analysis (FMA-100) [8] which uses a scanned tunable laser source (TLS) and a charged coupled device (CCD) camera. The TLS is scanned across the measurement bandwidth and the 2D spatial intensity profile at the output of the FUT is collected by the CCD as a function of wavelength. The system can take a measurement in $<1$ minute [8]. Whilst fast, such a response time is still unsatisfactory for a number of practical applications, for instance when the coupling into the FUT is subject to changes (random or intentional), or again when monitoring MMF interconnections (e.g. splices) or devices (e.g. long period grating mode converters) during their manufacture [9]. All these examples require the ability to carry out $\mathrm{S}^{2}$ measurements in real time, something that has never been reported to date.

In this paper, we demonstrate applications of an improved system implementation of the $S^{2}$ technique [10], which has an 
increased repetition speed enabling the ability to measure in real time $(\sim 1 \mathrm{~s})$. This is achieved by sweeping the TLS source across the target wavelength range, directly and synchronously triggering a high sensitivity InGaAs CCD camera to record intensity profile images every $\sim 2 \mathrm{~ms}$, whilst processing the data inline. Wavelength sweeping is obviously well documented in applications such as spectroscopy [11] and SDM system characterization [12], but to the best of our knowledge has never been demonstrated for real-time $S^{2}$ measurements. In the following, we describe the system and data collection in detail, we demonstrate its performance improvement by comparing it to the standard stepped $\mathrm{S}^{2}$ method, and finally we present the fast acquisition of a $S^{2}$ spectrogram and a detailed real-time investigation of the polarization dependency of modal excitation in a hollow-core photonic bandgap fiber (HC-PBGF), as two practical demonstrations of the system.

\section{REAL TIME $\mathrm{S}^{2}$ IMAGING}

Our wavelength-swept system (Fig. 1) is based on a high specification TLS (Agilent $81940 \mathrm{~A}$ ) with $100 \mathrm{kHz}$ nominal linewidth, which is swept at a rate of $500 \mathrm{pm} / \mathrm{s}$ over the $1520-1630 \mathrm{~nm}$ wavelength range. The output from the FUT, after the usual magnification (and optional polarization control) stages, is imaged via an InGaAs camera (Xenics Xeva $-1.7-320$ ) with a $320 \times 256$ pixel focal plane array with $30 \mu \mathrm{m}$ pixel pitch and $100 \mathrm{~Hz}$ nominal frame rate, which can be further increased to $500 \mathrm{~Hz}$ by selecting a reduced size window of interest (64x64 pixels). During each wavelength sweep the TLS is set to produce a hardware high frequency triggering signal which is fed to the camera to indicate when to acquire a frame. This approach allows the reliable operation of the camera at the maximum windowed frame rate. To be able to provide a real time measurement capability, both the data collection and the necessary data processing need to occur within a maximum approximate timescale of $\sim 1 \mathrm{~s}$. When operated in wavelength swept mode, our TLS source has a $\approx 0.22$ s run up/run down time between two successive scans, which we reduced from 6s [10] by multi-sweeping the TLS (a 27 times improvement). To provide a wavelength resolution of $\approx 1 \mathrm{pm}$, generally adequate for most measurements and able to accommodate modal delays up to $4 \mathrm{~ns}$, we set it to provide 400 triggers (one every $2 \mathrm{~ms}$ ) during a $\approx 0.8$ s measurement time, in such a way to match the $500 \mathrm{~Hz}$ frame repetition rate of the camera. Thus, a repeatable full sweep scan and data collection takes just over one second $(\sim 1.02 \mathrm{~s})$. Note that the TLS triggering method ensures that no data is collected while the TLS is getting up to the sweep speed [13]. Furthermore, the system is able to correct for any scan speed inconsistencies within a single wavelength scan, as the wavelength at which the trigger is sent can be dynamically measured in the Keysight 81940A TLS used. The sweeping approach affords an $\approx x 100$ (at least) improvement in measurement speed as compared to the equivalent system operated in a stepped wavelength mode [14]. The techniques are broadly similar except that the TLS is stepped to each wavelength rather than using a continuous sweep. The stepping process is repeated until all the wavelengths are collected. It should also be noted that the measurement speed of our system is largely limited by the frame rate of the camera - by using one of the latest generation fast near-IR cameras currently available on the market we anticipate further significant gains in terms of scan speed (e.g. an $\approx 20 x$ improvement by using $1730 \mathrm{~Hz}$ frame rate CCD windowed to $10 \mathrm{kHz},[15])$.

We also implemented an inline data processing system, which seamlessly receives data from the camera, performs all the necessary post-processing [7] and provides the results of the $\mathrm{S}^{2}$ measurement in $\sim 0.3 \mathrm{~s}$, as shown in Fig.1 (a)(inset). The CCD is interfaced to a PC via a Labview user interface loop. The inline processing is achieved using a pipeline architecture, where each data analysis task is broken down into sections and the data is passed from one section to the next. This allows the regular stream of individual wavelength dependent images to be collected into each measurement dataset and processed seamlessly, without waiting for the previous dataset to be analyzed. The system is able to perform a single measurement, i.e. data collection $(0.8 \mathrm{~s})$ and processing $(0.3 \mathrm{~s})$, in just over one second ( 1.1s). Our system provides in real time, the DGD plot, MPI value, intensity profile and relative phase profile of any higher order mode (HOM). Thanks to the use of sampling correction techniques discussed in reference [16], its MPI values are accurate to within $\approx 1 \mathrm{~dB}$.

\section{REDUCED NOISE FLOOR VIA DATA SET AVERAGING}

Fig. 2 shows a direct comparison between a wavelengthswept and a more conventional wavelength-stepped $\mathrm{S}^{2}$ measurement taken with identical measurement parameters (1550-1558nm, 20pm resolution, 401 wavelengths) on a standard single mode fiber (SSMF). The measurements took $0.8 \mathrm{~s}$ and $420 \mathrm{~s}$ for the swept and stepped method, respectively. We chose to measure an SMF, which generates largely featureless DGD plots, as this allows a direct comparison of the relative background noise levels, which, in the more general case of a MMF, ultimately determines the ability to discern peaks. The two noise floors are determined mainly by the scan parameters, and indeed they appear very similar for the two measurements at 50-120ps delays. On the other hand, beyond $150 \mathrm{ps}$, the wavelength stepped trace shows a higher level and oscillations of the noise floor, which we ascribe to variations in the source intensity, CCD response and MMF coupling conditions over the time required to perform the measurement. Furthermore, as the swept measurement is about 500x faster, it is possible to collect and average $N$ datasets, and process the results in the standard way. The resulting improvement in the signal to noise ratio at a rate of $\sqrt{N}$ [17] produces a reduction of the MPI noise floor proportional to $N$.

This is shown by the orange trace in Fig. 2, which is obtained by averaging 10 wavelength-swept datasets $(\approx 12.4 \mathrm{~s}$ total collection time) and achieves $\approx 9.8 \mathrm{~dB}$ increase in the MPI sensitivity, as compared to a single swept measurement. Using the same approach and higher averaging a reliable noise floor down to $\approx-70 \mathrm{~dB}$ can be achieved for multimode fiber with our 12 bit CCD, which could further be improved with a 14 bit CCD [15]. The features observed in the region below $25 \mathrm{ps}$ of the 


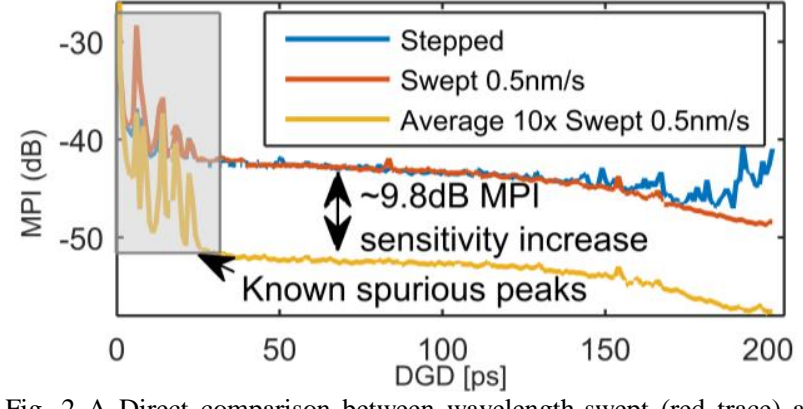

Fig. 2 A Direct comparison between wavelength-swept (red trace) and wavelength-stepped (blue trace) $S^{2}$ measurements on a SSMF. The orange curve shows the DGD plot obtained by averaging 10 swept measurements and shows substantial MPI sensitivity increase, $\sim 9.8 \mathrm{~dB}$. SMF length is $\sim 9.5 \mathrm{~m}$.

DGD plot do not correspond to real modal peaks but are due to replicas of the FM, originating from well identified reflections from various optical elements within the setup (e.g. lenses, polarizers and a sapphire window built in the CCD array) [10]. Nevertheless, they illustrate the large increase in sensitivity of the wavelength swept method when used in association with averaging. As it can be seen, by reducing the measured noise, averaging gives a more accurate reading of the true MPI of a mode, which for peaks close to the measured floor can be significantly lower than estimated from a single measurement.

\section{High RESOLUTION $S^{2}$ SPECTROGRAM}

We now present two practical examples, in which the system described here, can benefit specific measurement problems. The first is the collection of $S^{2}$ spectrograms, i.e. $2 \mathrm{D}$ plots showing how the MPI of each of the modes in a MMF varies as a function of DGD and wavelength, which was first introduced by Jasapara and Yablon [18]. This tool is available commercially from Interfiber Analysis (FMA-100) [8] and measures around the $1050 \mathrm{~nm}$ or $1550 \mathrm{~nm}$ regimes. A standard spectrogram is more frequently obtained through use of a stationary fiber probe at the MMF output coupled in to a single detector (rather than using a camera) to save acquisition and processing time. In this case, however, the information on the spatial intensity distribution of the modes is lost. In contrast, our improved technique allows measurement over very wide wavelength intervals, and over timeframes for which environmental fluctuations are less likely to perturb the measurement. Fig. 3(a) shows the $S^{2}$ spectrogram over the full range (1520-1630nm) of our TLS, in 1pm steps and at $64 \times 64$ spatial resolution, collecting a total of 110,000 frames. Fig. 3(b), modal images are generated from the DGD values highlighted in Fig. 3(c), a subset of the $S^{2}$ spectrogram, between $1555 \mathrm{~nm}$ and $1560 \mathrm{~nm}$. The FUT in this case is a 19 cell low loss wide bandwidth HC-PBGF reported in a previous work [20], looking in particular at its $160 \mathrm{~nm}$ wide, surface mode free, low transmission loss window. The differential dispersion curves of several high order modes can be clearly identified and immediately linked to the image of the mode that generated it. The full measurement took less than $600 \mathrm{~s}$, as compared with the $\sim 6$ hours and $45 \mathrm{mins}$ that would have been necessary to collect the data using a conventional wavelength stepped system.

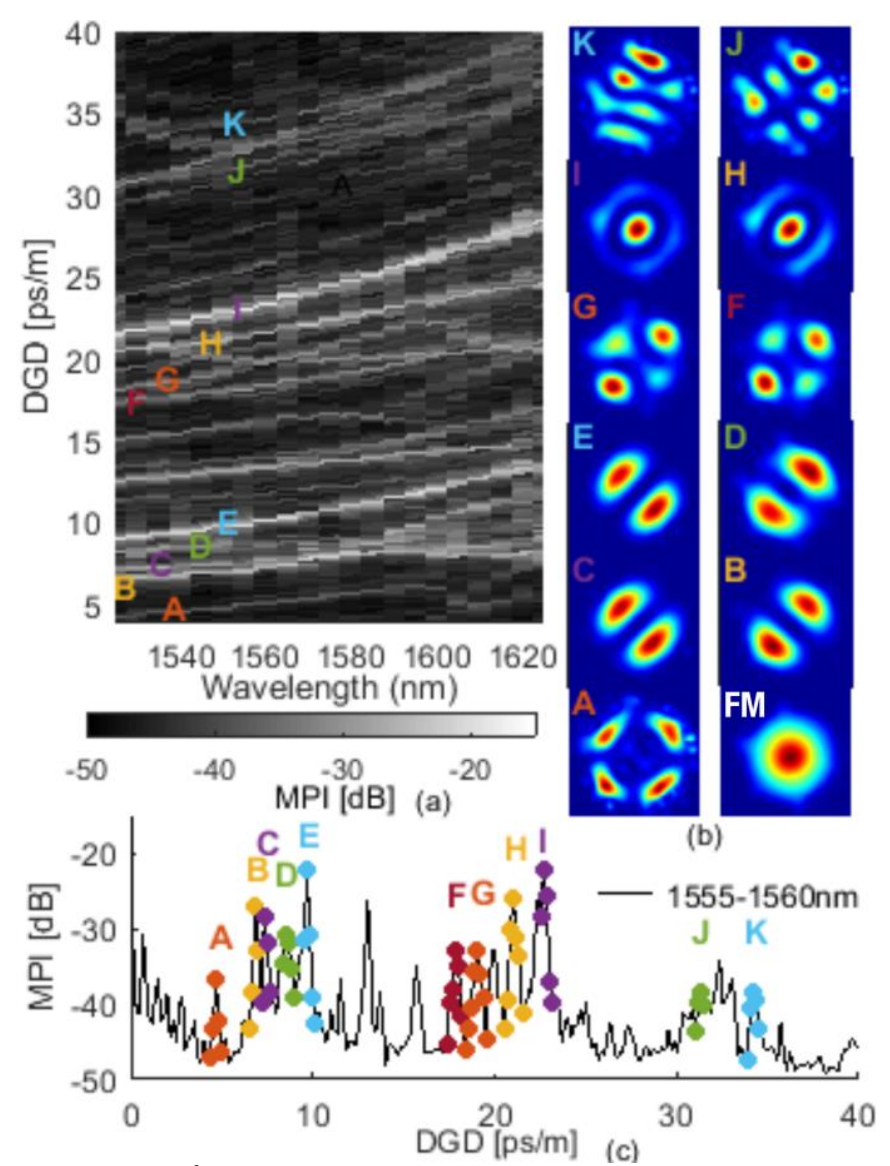

Fig. $\underline{3}$ (a) $S^{2}$ Spectrogram of a HC-PBGF [19] obtained using the fast wavelength-swept $S^{2}$ technique with $1 \mathrm{pm}$ resolution over $1520-1630 \mathrm{~nm}$. The chosen wavelength bandwidth was $5 \mathrm{~nm}$ for the data processing and was stepped along at $5 \mathrm{~nm}$ steps. (b) Modal images within this fiber. (c), modal DGD peaks measure between $1555-1560 \mathrm{~nm}$. Modal images in (b) are calculated from values highlighted with dots in (c).

\section{REAL-TIME MODAL MONITORING}

The second example is the real-time monitoring of launch conditions in a multimode fiber, for which again we used the same HC-PBGF mentioned above. From previously published data [19], and from the $S^{2}$ spectrogram above, this fiber is able to guide 4-5 mode groups, which can be selectively excited, e.g. by slightly offsetting the launch and varying the polarization. In this instance we concentrate on tracking in real time the relative power of the 4 modes forming the $\mathrm{LP}_{11}$-like group as the input polarization is rotated. In our experiment, the polarization was rotated at a constant rate of $24.4 \mathrm{mrad} / \mathrm{s}$ using a half-waveplate mounted on a motorized stage. This polarization control was situated in the collimated beam path between two matched lenses which launched power from a SMF into the FUT with a slight offset to deliberately excite the HOM content. $\mathrm{S}^{2}$ measurements were taken in the range $1550-1558 \mathrm{~nm}$ with $20 \mathrm{pm}$ resolution and a sweep speed of $10 \mathrm{~nm} / \mathrm{s}$, producing a measurement every $1.24 \mathrm{~s}$. The MPI and DGD values for the four peaks corresponding to the $\mathrm{LP}_{11}$-like modes obtained from DGD plots, corrected for spectral leakage and sampling errors [16], and plotted as a function of time, are shown in Fig. 4. Each point in Fig. 4(c) represents a separate measurement. As can be seen, the MPI of modes A and D (same polarization, different 

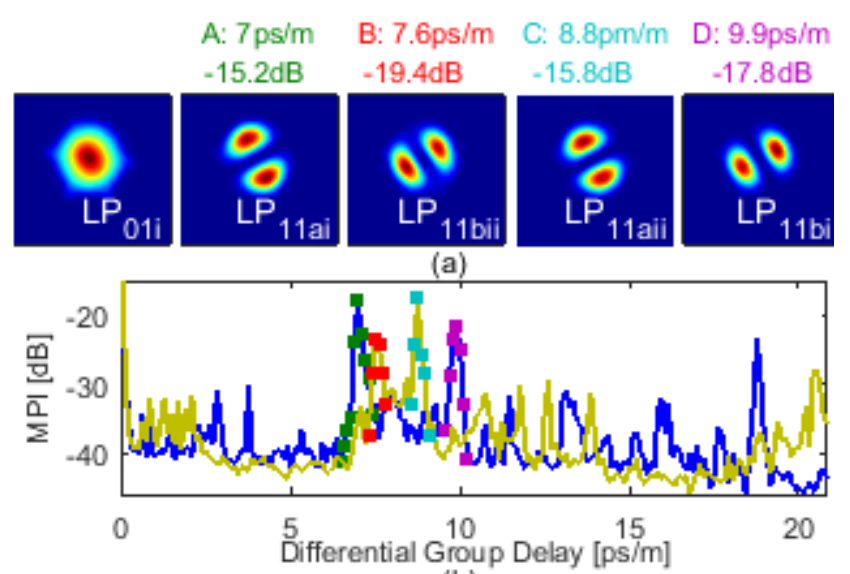

(b)

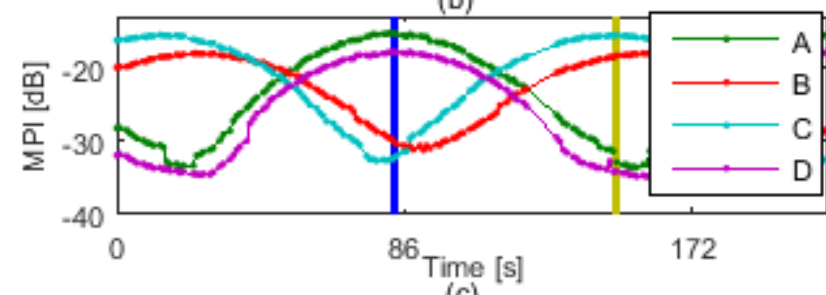

(c)

Fig. 4. A real-time $S^{2}$ measurement of the modal content of a 19 cell HC-PBGF [19], showing: (a) the modal images of $\mathrm{LP}_{01}, \mathrm{LP}_{11}$, (b) relative DGD \& MPI and (c) MPI vs. time as the polarization angle is rotated at a constant rate of $24.4 \mathrm{mrad} / \mathrm{s}$.

spatial orientation) is in antiphase with that of modes B and C (opposite polarization). The amount of power in each of the four $\mathrm{LP}_{11}$ modes periodically changes with the angle of rotation, reaching MPI levels in the range $\sim 15-35 \mathrm{~dB}$ and showing the expected periodicity. This example shows how real-time monitoring could be used, for example, to optimize coupling/splicing to a MMF and how it could assist the fabrication of other MMF-based optical devices.

\section{CONCLUSION}

In conclusion, we have presented a much improved system implementation of the $\mathrm{S}^{2}$ imaging technique providing a real-time measurement capability, which we believe represents a step change in the usefulness of this technique to support the development of MMF and related fiber devices. The technique exploits continuous wavelength-sweeping of a tunable laser source in combination with direct synchronous triggering of a CCD camera. It is as fast as another real time modal characterization technique recently reported, $C^{2}$ [13] which however requires the added complexity of establishing a proper two path optical interferometer to operate. The set-up reported in this work offers a real time data analysis capability, i.e. an $\mathrm{S}^{2}$ measurement is collected, and its result produced, in $\sim 1 \mathrm{~s}$. The speed increase can be exploited e.g. to dramatically reduce the measurement noise floor over the full measured DGD, thereby increasing the sensitivity and MPI determination accuracy, by collecting and averaging multiple measurements. Another key demonstrated capability is to collect the underlying data for wide bandwidth $S^{2}$ spectrograms in $\approx$ minutes. Finally, the ability to monitor the modal content of a MMF as a function, e.g. of input coupling, opens up the possibility of online monitoring, e.g. of splices, couplers and other devices whilst they are fabricated, which will provide an invaluable tool for future development of MMF technologies. We anticipate that further improvements may well be possible by reducing the idle time of the TLS and using a faster camera.

\section{ACKNOWLEDGMENT}

F. Poletti and D. J. Richardson kindly acknowledge support from the Royal Society. D. R. Gray kindly acknowledges software development support from Clayton Peters. The data for this work is accessible through the University of Southampton Institutional Research Repository (DOI:10.5258/SOTON/379582)

\section{REFERENCES}

[1] V.A.J.M. Sleiffer. et. al.. "High capacity mode-division multiplexed optical transmission in a novel 37-cell hollow-core photonic bandgap fiber," J. LightwaveTechnol. 32(4) , pp. 854-863. (2014, Feb).

[2] U. Griebner, R. Grunwald, R. Koch, and H. Schönnagel. "Efficient laser operation with nearly diffraction-limited output from a diode-pumped heavily Nd-doped multimode fiber. Opt. Lett. 21, pp. 266-268 (1996).

[3] C. Belleville and G. Duplain, "White-light interferometric multimode fiber-optic strain sensor," Opt.. Lett. 18, pp. 78-80 (1993).

[4] M. E. Fermann, "Single-mode excitation of multimode fibers with ultrashort pulses,", Opt. Lett., 23, pp. 52-54 (1998).

[5] P. S. Szczepanek and J. W. Berthold, "Side launch excitation of selected modes in graded-index optical fibers," Appl. Opt., 17, pp. 3245-3247 (1978).

[6] M. Paurisse, et. al., "Complete measurement of fiber modal content by wavefront analysis," Opt. Express, 20, pp. 4074-4084, (2012).

[7] J. W. Nicholson, A. D. Yablon, S. Ramachandran, and S. Ghalmi, "Spatially and spectrally resolved imaging of modal content in large-mode-area fibers," Opt. Express 16(10), pp. $7233-7243$ (2008).

[8] Interfibre Analysis, "FMA-100", http://www.interfiberanalysis.com/images/FMA100 datasheet Sept201 3.pdf.

[9] I. Giles, et. al., "Fiber LPG Mode Converters and Mode Selection Technique for Multimode SDM," IEEE Photon. Technol. Lett. 24(21), pp. 1922-1925 (2012).

[10] D. R. Gray, et. al., "Towards Real-Time Mode Content Characterization of Multimode Fibers," Proceedings of European Conference on Optical Communication (ECOC) (IEEE, 2014), paper Th.1.4.3.

[11] K. G. Libbrecht and, M. W. Libbrecht, "Interferometric measurement of the resonant absorption and refractive index in rubidium gas," Am. Journal of Phys., 74, pp. 1055-1060 (2006).

[12] N K. Fontaine, "Characterization of multi-mode fibers and devices for MIMO communications," Proc. SPIE 9009, 90090A (2013).

[13] J. Demas and S. Ramachandran, "Sub-second mode measurement of fibers using C ${ }^{2}$ imaging," Opt. Express 22, pp. 23043-23056 (2014).

[14] S. Blin, et. al., "Simple Modal Analysis Method for Multi-Mode Fibers," Proceedings of European Conference on Optical Communication (ECOC) (IEEE, 2009), paper P1.16.

[15] Xenics, "Cheetah-640CL http://www.xenics.com/en/cheetah-640cl.

[16] D. R. Gray, et. al., "Accurate calibration of $S^{2}$ and interferometry based multimode fiber characterization techniques," Opt. Express, 23(8), pp. 10540-10552, (2015).

[17] R. Hui and M. O'Sullivan, "Fiber Optic Measurement Techniques" (Academic, 2009), p391.

[18] J. Jasapara and A. D. Yablon, "Spectrogram approach to S2 fiber mode analysis to distinguish between dispersion and distributed scattering," Opt. Lett. 37, pp. 18 (2012).

[19] N. V. Wheeler, et.al., "Wide-bandwidth, low-loss, 19-cell hollow core photonic band gap fiber and its potential for low latency data transmission," Optical Fiber Communication Conference, (Optical Society of America, 2012), paper PDP5A.2. 